\title{
Cerebellum: from Fundamentals to Translational Approaches. The Seventh International Symposium of the Society for Research on the Cerebellum
}

\author{
Mario Manto $^{1}$ - Peter Mariën ${ }^{2,3}$
}

Published online: 7 January 2016

(C) Springer Science+Business Media New York 2016

\begin{abstract}
In terms of cerebellar research and ataxiology, a most fascinating period is currently going on. Numerous academic groups are now focusing their innovative research on the so-called little brain, hidden at the bottom of our brain. Indeed, its unique anatomical features make the cerebellum a wonderful window to address major questions about the central nervous system. The seventh international symposium of the SRC was held in Brussels at the Palace of Academies from May 8 to 10, 2015. The main goal of this dense symposium was to gather in a 2-day meeting senior researchers of exceptional scientific quality and talented junior scientists from all over the world working in the multidisciplinary field of cerebellar research. Fundamental and clinical researchers shared the latest knowledge and developments in this rapidly growing field. New ideas, addressed in a variety of inspiring talks, provoked a vivid debate. Advances in genetics, development, electrophysiology, neuroimaging, neurocognition and affect, as well as in the cerebellar ataxias and the controversies on the roles and functions of the cerebellum were presented. The Ferdinando Rossi lecture and the key-note lecture were delivered by Jan Voogd and Chris De Zeeuw, respectively. Contacts between researchers of different neuroscientific
\end{abstract}

Mario Manto

mmanto@ulb.ac.be

1 Unité d'Etude du Mouvement (UEM), FNRS, ULB-Erasme, 808 Route de Lennik, 1070 Brussels, Belgium

2 Clinical and Experimental Neurolinguistics, CLIN, Vrije Universiteit Brussel, Brussels, Belgium

3 Department of Neurology and Memory Clinic, ZNA Middelheim Hospital, Antwerp, Belgium disciplines established a robust basis for novel trends and promising new cooperations between researchers and their centers spread all over the world.

Keywords Cerebellum $\cdot$ Research $\cdot$ Ataxias $\cdot$ Fundamental . Translational $\cdot$ Clinical

The seventh international symposium of SRC was held in Brussels from May 8 to 10, 2015 at the prestigious Palace of the Academies, which was built between 1823 and 1828 for Prince William of Orange and which currently functions as the seat of the Royal Academies of for Science and the Arts of Belgium. During two intense days, eminent researchers from many countries presented their work and their main findings in seven thematic sessions, in a unique and friendly atmosphere inherent of the SRC symposia (Fig. 1). Genetic and developmental issues, electrophysiological aspects, advances in neuroimaging of the cerebellum, cerebellar disorders (ataxias and essential tremor), neurocognitive, affective and social impacts of cerebellar pathology, and therapeutic approaches to cerebellar disease and controversies were addressed in detail. The organizing committee of the seventh international symposium and the executive committee of the SRC are particularly grateful to all the chairmen and speakers who made of this symposium held in the center of Europe an unforgettable, inspiring and highly successful event. The support of the sponsors (FNRS, FWO, BioMed Central, Springer, VisitBrussels) is also gratefully acknowledged. This editorial briefly summarizes the scientific topics that were discussed during the 2-day symposium.

The cerebellum is particularly vulnerable to malformations and disruptions since it develops from the early embryonic period to the first postnatal years. In the first thematic session devoted to congenital and developmental aspects of the 


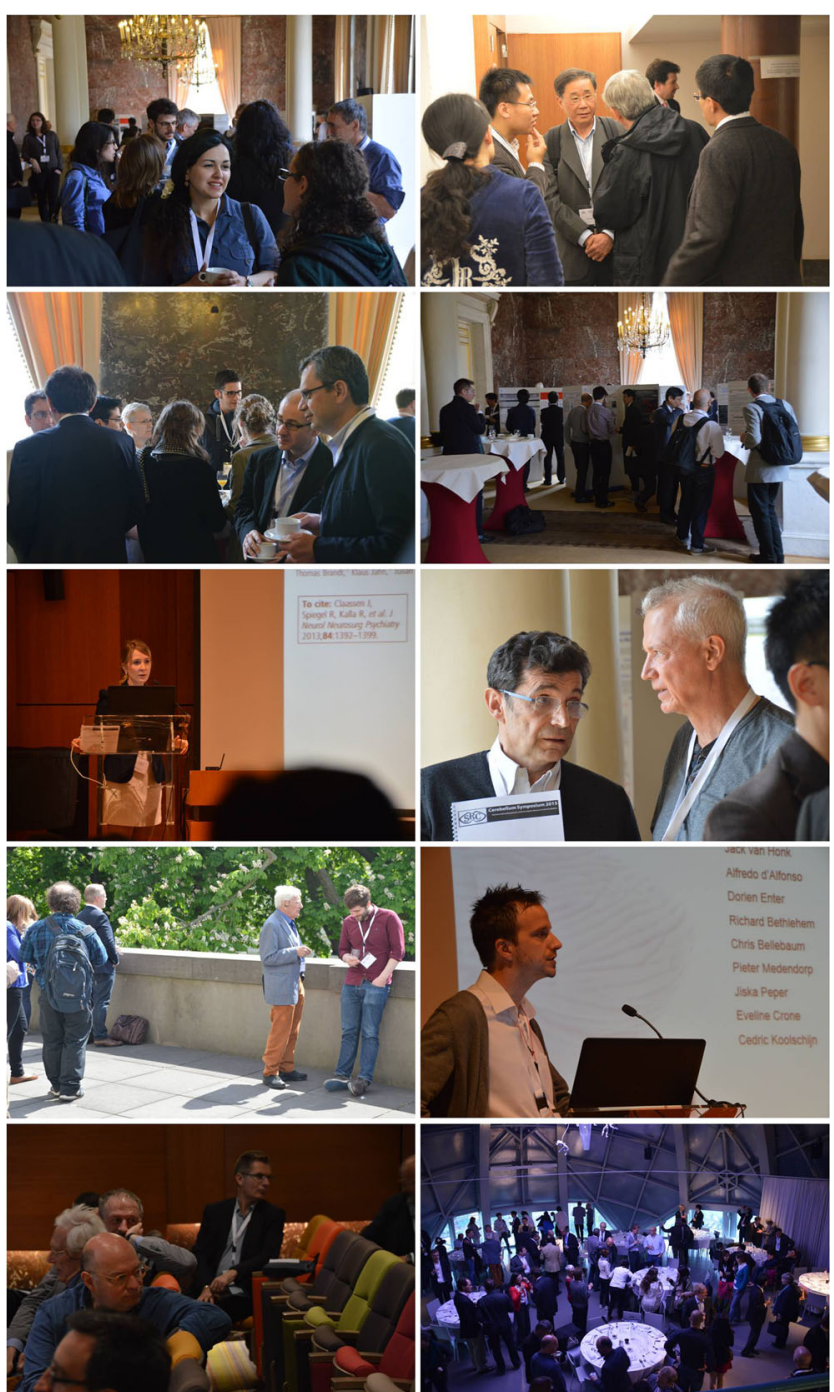

Fig. 1 Pictures of the attendees taken during the seventh symposium in Brussels, Belgium

cerebellum and cerebellar pathology, Roy Sillitoe addressed novel genetic mouse models that might become very relevant for cerebellar disorders. Nori Koibuchi subsequently discussed the important links between hormones and cerebellar development, with an emphasis on thyroid hormones and their physiological action. Andrea Poretti explained the critical importance of pre- and postnatal imaging in the diagnosis of congenital and developmental disorders of the cerebellum [1].

The second session of the symposium dealt with electrophysiological aspects of the cerebellum, from its cells to its networks. It is well established that the cerebellar nuclei play a central role because they represent the sole output of cerebellar circuitry. Eric Lang presented novel findings on the effects of complex spikes on cerebellar nuclei discharges [2]. Clear evidence was provided of synchrony as a mechanism by which complex spikes tune the electrophysiological patterns in cerebellar nuclei. In his talk, Egidio D'Angelo addressed the advances in our understanding of the electrophysiological properties of the cerebellar cortex. Abigail Person discussed the sensorimotor integrative mechanisms in the mammalian cerebellum. Finally, Chris De Zeeuw presented a talk focusing on the mechanisms of the plasticity of the cerebellar networks, starting from the anatomy to the electrophysiology.

Advances in the neuroimaging of the cerebellum were presented in the third thematic session. Anna Letizia Allegra Mascaro showed how researchers can take advantage of the optical techniques to assess the anatomy, the plasticity and various functions of the cerebellum [3]. Detailed 3D reconstructions of cerebellar axons represent an example of the recent advances made in this rapidly growing field of research. Dagmar Timmann presented state-of-the-art MRI methods to characterize structural alterations of the cerebellum [4]. These methods represent powerful diagnostic tools. Gülin $\mathrm{Oz}$ discussed the scope and limits of magnetic resonance spectroscopy (MRS) of the cerebellum. She demonstrated that MRS detects parallel neurochemical alterations in patients and mouse models of SCA prior to structural MRI changes and before overt pathology is observed.

The fourth session of the symposium was dedicated to cerebellar disorders. Nicolas Dupré presented an overview of the clinical, neurophysiological, genetic and neuroimaging characteristics of the autosomal recessive cerebellar ataxias. The non-ataxic presentations of adolescent or adult-onset dominant ataxias were reviewed by Elsdon Storey [5]. Both nervous system (visual symptoms, deafness, neuropathy, movement disorders, neuropsychiatric presentations) and non-nervous system features were discussed. Elan Louis provided a framework for the pathogenesis of essential tremor on the basis of recent findings showing a major involvement of the cerebellar cortex. To close the fourth session, Jerôme Honnorat discussed the fundamental mechanisms of the immune cerebellar ataxias.

The fifth session of the symposium addressed the role of the cerebellum in neurocognition, affect and social behaviour. Jeremy Schmahmann presented converging evidence from clinical, anatomical and functional neuroimaging studies demonstrating that the cerebellum is a major player in the modulation of a variety of cognitive and affective operations. The constellation of the neuropsychological and behavioralaffective deficits linked to cerebellar pathology is now coined Schmahmann's syndrome [6]. Dennis Schutter discussed the links between cerebellar circuitry and mood disorders [7]. He discussed the anatomical basis for a role of the cerebellum in reward and homeostatic regulation. Catherine Stoodley provided an overview of clinical and functional neuroimaging findings in a number of neurodevelopmental disorders in which the cerebellum is crucially implicated, including developmental dyslexia, attention deficit hyperactivity disorder (ADHD) and autism spectrum disorders (ASD) [8]. The 
disruption of specific cerebello-cerebral circuits in each disorder is now hypothesized.

The sixth session covered the field of therapeutic approaches to cerebellar pathology. A great need exists for effective medications in cerebellar ataxias in order to alleviate symptoms and improve the quality of life of the patients. Katharina Feil presented an update on the pharmacological treatments of cerebellar ataxias and nystagmus, in particular therapies with 4-aminopyridines (4-AP), acetazolamide, chlorzoxazone and acetyl-DL-leucine [9]. A number of ongoing randomized placebo-controlled trials were presented. Neuromodulation of the cerebellum by means of tDCS and TMS is a novel and steadily growing field in the therapeutic approaches to cerebellar pathology. Alberto Priori showed how the activity of the cerebellum can be modulated noninvasively with the goal of improving levodopa-induced dyskinesias in Parkinson's disease [10]. As highlighted by Jan Cendelin, great hope exists that neurotransplantations will soon represent a viable therapeutical strategy. Studies in mutant mice are very encouraging and further experiments are needed to jump successfully to human trials in the near future [11]. Bing-wen Soong discussed in his talk the dominant ataxias with a focus on TGM6 as a novel causative gene of SCA35 and its clinical features. SCA35 was identified as a relatively rare subtype of ADCA, clinically characterized by cerebellar ataxia, corticospinal tract signs, hand tremors and EPS. The perturbation of the protein stability and transglutaminase activity, as well as sensitisation of cells to apoptosis by increasing the activities of caspases were identified as the pathomechanisms underlying SCA35.

The seventh and last session of the symposium was dedicated to controversies. In the first part, Tomoo Hirano discussed the plasticity of cerebellar circuits and demonstrated that long-term depression (LTD) and rebound potentiation (RP) represent two mechanisms that might be active and used differently in a variety of motor tasks [12]. In his talk entitled 'Channelopathies: from genes to mouse models to mechanisms', Arn van den Maagdenberg discussed the involvement of the cerebellum in channelopathies including migraine. In the second part of the controversy section, Tim Ebner dealt with the beam versus radial organization of the cerebellum while Marco Molinari discussed the issue of the uniform computation/function of the cerebellum. These presentations unambiguously demonstrated that critical questions on the physiological and pathophysiological roles of the cerebellum remain a matter of vivid debate for the whole scientific community.

In memory of our friend and colleague Ferdinando Rossi, Jan Voogd delivered the first Ferdinando Rossi lecture on Deiters nucleus [13]. Voogd underlined the broad range of the wonderful works of Deiters on the lateral vestibular nucleus which receives information from the lateral vermal B zone and which is the origin of the lateral vestibulospinal tract.
According to Voogd, Deiters nucleus should be considered a cerebellar nucleus.

As highlighted by Tim Ebner in his introduction, the keynote lecture at the symposium was offered to Chris De Zeeuw for his outstanding contributions to the field of cerebellar research during the last two decades. Novel insights into our understanding of the mechanisms of learning and plasticity were put forward and discussed in-depth, opening novel doors for cerebellar research in the coming decades.

In two oral sessions covering 57 poster presentations, young scientists briefly summarized and presented the main findings of their research. The SRC also nominated the first Fellows of SRC for their outstanding services to the society: Maria Teresa Viscomi (Italy), Shoji Tsuji (Japan), Kazuyoshi Tsutsui (Japan), Yoshikazu Ugawa (Japan), Dagmar Timmann (Germany) and Jérôme Honnorat (France). The Masao Ito awards for the best posters were announced by our President Jean Mariani. The recipients were Oostland M. (Edinburgh, UK) for a poster dedicated to the roles of HCN1 (hyperpolarization-activated cyclic nucleotide-gated channel 1) channels in the inferior olive in motor learning, Rasmussen A. (Lund, Sweden) for a work on inhibition of the inferior olive during eyeblink conditioning, and Ferrari C. (Pavia, Italy) for a study showing that the cerebellum is involved in predictions in the cognitive domain. Our President concluded this successful symposium.

The atmosphere during the symposium was very friendly and scientifically highly inspiring, leading to novel collaborations amongst research groups across countries and continents. We now look very much forward to meeting you all again at the next SRC meeting in Winnipeg, Manitoba (Canada) from May 28 to 30, 2017 to enjoy scientifically exceptional talks and share the unique and friendly atmosphere of our symposia!

\section{References}

1. Poretti A, Boltshauser E, Huisman TA. Pre- and postnatal neuroimaging of congenital cerebellar abnormalities. Cerebellum. 2015.

2. Tang T, Suh CY, Blenkinsop TA, Lang EJ. Synchrony is key: complex spike inhibition of the deep cerebellar nuclei. Cerebellum. 2015.

3. Allegra Mascaro AL, Sacconi L, Silvestri L, Knott G, Pavone FS. Multi-modal optical imaging of the cerebellum in animals. Cerebellum. 2015.

4. Deistung A, Stefanescu MR, Ernst TM, Schlamann M, Ladd ME, Reichenbach JR, Timmann D. Structural and functional magnetic resonance imaging of the cerebellum: considerations for assessing cerebellar ataxias. Cerebellum. 2015.

5. Storey E. Non-ataxic presenting symptoms of dominant ataxias. Cerebellum. 2015.

6. Manto M, Mariën P. Schmahmann's syndrome - identification of the third cornerstone of clinical ataxiology. Cerebellum Ataxias. $2015 ; 2: 2$. 
7. Schutter DJ. A cerebellar framework for predictive coding and homeostatic regulation in depressive disorder. Cerebellum. 2015.

8. Stoodley CJ. The cerebellum and neurodevelopmental disorders. Cerebellum. 2015.

9. Feil K, Bremova T, Muth C, Schniepp R, Teufel J, Strupp M. Update on the pharmacotherapy of cerebellar ataxia and nystagmus. Cerebellum. 2015.

10. Ferrucci R, Cortese F, Bianchi M, Pittera D, Turrone R, Bocci T, Borroni B, Vergari M, Cogiamanian F, Ardolino G, Di Fonzo A,
Padovani A, Priori A. Cerebellar and motor cortical transcranial stimulation decrease levodopa-induced dyskinesias in Parkinson's disease. Cerebellum. 2015.

11. Cendelin J. Transplantation and stem cell therapy for cerebellar degenerations. Cerebellum. 2015.

12. Hirano T, Yamazaki Y, Nakamura Y. LTD, RP, and motor learning. Cerebellum. 2015.

13. Voogd J. Deiters' nucleus. Its role in cerebellar ideogenesis: the Ferdinando Rossi memorial lecture. Cerebellum. 2015. 